\title{
Accessory auricle: Classification according to location, protrusion pattern and body shape
}

\author{
Jungil Hwang, , Jaeyoung $\mathrm{Cho}^{2}$, Jin Sik Burm ${ }^{1}$ \\ ${ }^{1}$ Department of Plastic Surgery, Kyung Hee University Medical Center, Kyung Hee University School of Medicine, Seoul; ${ }^{2}$ Department of \\ Plastic Surgery, Kyung Hee University Hospital at Gangdong, Kyung Hee University School of Medicine, Seoul, Korea
}

Background Accessory auricles (AAs) are common congenital anomalies. We present a new classification according to location and shape, and propose a system for coding the classifications.

Methods This study was conducted by reviewing the records of 502 patients who underwent surgery for AA. AAs were classified into three anatomical types: intraauricular, preauricular, and buccal. Intraauricular AAs were divided into three subtypes: intracrural, intratragal, and intralobal. Preauricular AAs were divided into five subtypes: precrural, superior pretragal, middle pretragal, inferior pretragal, and prelobal. Buccal AAs were divided into two subtypes: anterior buccal and posterior buccal. AAs were also classified according to their protrusion pattern above the surrounding surface: pedunculated, sessile, areolar, remnant, and depressed. Pedunculated and sessile AAs were subclassified as spherical, ovoid, lobed, and nodular, according to their body shape. Cartilage root presence and family history of AA were reviewed. A coding system for these classifications was also proposed.

Results The total number of AAs in the 502 patients was 1,003. Among the locations, the superior pretragal subtype (27.6\%) was the most common. Among the protrusion patterns and shapes, pedunculated ovoid AAs were the most common in the preauricular $(27.8 \%)$ and buccal areas (28.0\%), and sessile lobed AAs were the most common in the intraauricular area (48.7\%). The proportion of AAs with a cartilage root was 78.4\%, and $11 \%$ of patients had a family history. The most common type of preauricular AA was the superior pretragal pedunculated ovoid AA (13.2\%) with a cartilage root.

Conclusions This new system will serve as a guideline for classifying and coding AAs.

Keywords Classification / Ear abnormalities / Embryology / Accessory auricle / Accessory tragus
Correspondence: Jin Sik Burm Department of Plastic Surgery, Kyung Hee University Medical Center, 23 Kyungheedae-ro, Dongdaemun-gu, Seoul 02447, Korea

Tel: +82-2-958-8431

Fax: +82-2-963-5638

E-mail: jsburm@gmail.com

Received: 3 May $2018 \bullet$ Revised: 6 Jul $2018 \bullet$ Accepted: 29 Aug 2018

pISSN: 2234-6163 • elSSN: 2234-6171 • https://doi.org/10.5999/aps.2018.00430 • Arch Plast Surg 2018;45:411-417

This study was presented at the 5 th Research and Reconstructive Forum of the Korean Society of Plastic and Reconstructive Surgeons on May 14-16, 2015, in Gangwon Province, Korea.

This study was presented at the 28th Annual European Association of Plastic Surgeons Meeting on May 25-27, 2017, in Pisa, Italy.

\section{INTRODUCTION}

Accessory auricles (AAs) are abnormal auricular appendages remaining after the formation of the anterior auricle in early embryonic development. External ears develop from the first and second pharyngeal arches during the 4th week of gestation. The first pharyngeal arch forms three hillocks, which fuse to form the anterior auricular structures. They are located at the side of the mouth and gradually move laterally and dorsally during development $[1,2]$. The failure of fusion of the three hillocks of 


\section{Fig. 1. Accessory auricles within a curved triangle}

Accessory auricles are found within a curved triangle from the oral commissure groove to the anterior auricle between the helical spine and the ear lobe.

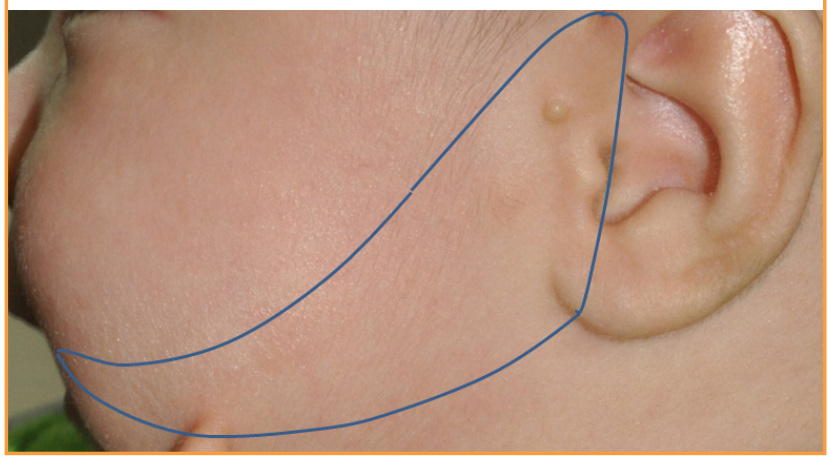

the first pharyngeal arch is thought to be the reason for the development of AA $[3,4]$. Their location can be anywhere along the migration tract within a curved triangle from the oral commissure groove to the anterior auricle between the helical spine and the ear lobe. Clinically, we have observed AAs within this curved triangle (Fig. 1).

Several studies have described these abnormal skin appendages as accessory tragi $[3,4]$ or preauricular tags $[5,6]$. However, these skin appendages may be present at the buccal, intra-auricular, or precrural areas, all of which are distinct from the preauricular area. They can also be present at the prelobal, crural, or lobal areas, none of which is in the tragal area. Thus, we propose that $\mathrm{AA}$ is the most proper term for these abnormal skin appendages.

AAs are common congenital anomalies with an incidence of 5 to 10 per 1,000 live births [5-7], and have various locations and shapes. Several studies have reported AA cases and described their features; however, they reported small numbers of cases [3-7]. In addition, few reports contain a classification scheme for AAs and a detailed description of AAs. Our center has performed hundreds of operations for AAs. We reviewed the medical records and photographs of such cases. The purpose of this report is to present a classification scheme for AAs according to location, protrusion pattern, and shape. Based on this classification, we developed a coding system for describing AAs. We expect that this new classification and coding system could provide guidance for the management of AAs.

\section{METHODS}

We retrospectively reviewed the medical records of 502 patients who underwent excision of AAs between January 2014 and March 2016. Patient demographics, family history of AA, photographs, and operative findings, including the presence and di-

\section{Fig. 2. Classification of AAs according to location}

Accessory auricles (AAs) were divided into three types: as intraauricular, preauricular, and buccal. The intraauricular unit was subdivided into three vertical auricular subunits: crural, tragal, and earlobal. The preauricular unit was subdivided into five vertical auricular subunits: crural, superior tragal, middle tragal, inferior tragal, and earlobal. The buccal unit was subdivided into two horizontal subunits: anterior buccal and posterior buccal. IC, intracrural; IT, intratragal; IL, intralobal; PC, precrural; PTs, superior pretragal; PTm, middle pretragal; $\mathrm{PTi}$, inferior pretragal; $\mathrm{PL}$, prelobal; $\mathrm{Ba}$, anterior buccal; Bp, posterior buccal.

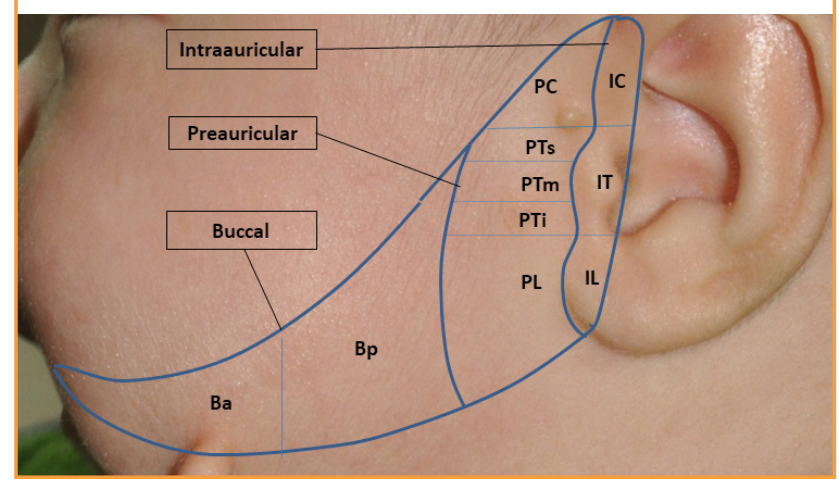

rection of a cartilage root, were obtained from medical records. Herein, we classify AAs according to their anatomical location, protrusion pattern, and body shape, and propose a coding system for these classifications.

\section{Classification according to location}

AAs were classified into two categories according to the facial anatomical unit and the auricular subunit. AAs were divided into three types - intraauricular, preauricular, and buccal-according to the horizontal location of the facial anatomical units (Fig. 2). The intraauricular unit invaded the auricular structures through the cartilage root or pedicle of the AA and was located posterior to the preauricular groove. The preauricular unit did not invade the auricular structures and was located anterior to the preauricular groove and posterior to the posterior border of the sideburns. The buccal unit was located on the cheek from the oral commissure groove to the sideburns.

The intraauricular unit was subdivided into three vertical auricular subunits: crural, tragal, and earlobal types. The preauricular unit was subdivided into five vertical auricular subunits: crural, superior tragal, middle tragal, inferior tragal, and earlobal types. The crural subunit was located above the inferior border of the interconchal ridge. The tragal subunit was located below the inferior border of the interconchal ridge to the intertragal notch. The earlobal subunit was located below the intertragal notch. The buccal unit was subdivided into two horizontal subunits - anterior buccal and posterior buccal — according to the vertical axis of the lateral canthus. 


\section{Fig. 3. Classification of AAs according to pattern and shape}

Accessory auricles (AAs) were classified as pedunculated, sessile, areolar, remnant, and depressed. Pedunculated and sessile patterns were subclassified as spherical, ovoid, lobed, and nodular according to their body shape. The pedunculated pattern had a neck and spherical, ovoid, or lobed shape. The sessile pattern did not have a neck and had a nodular, ovoid, or lobed shape. The areolar pattern was a pigmented and slightly convex lesion with a wide round shape, similar to the nipple areola. The remnant pattern was tiny and slightly protruding. The depressed pattern was a dimple or pit.

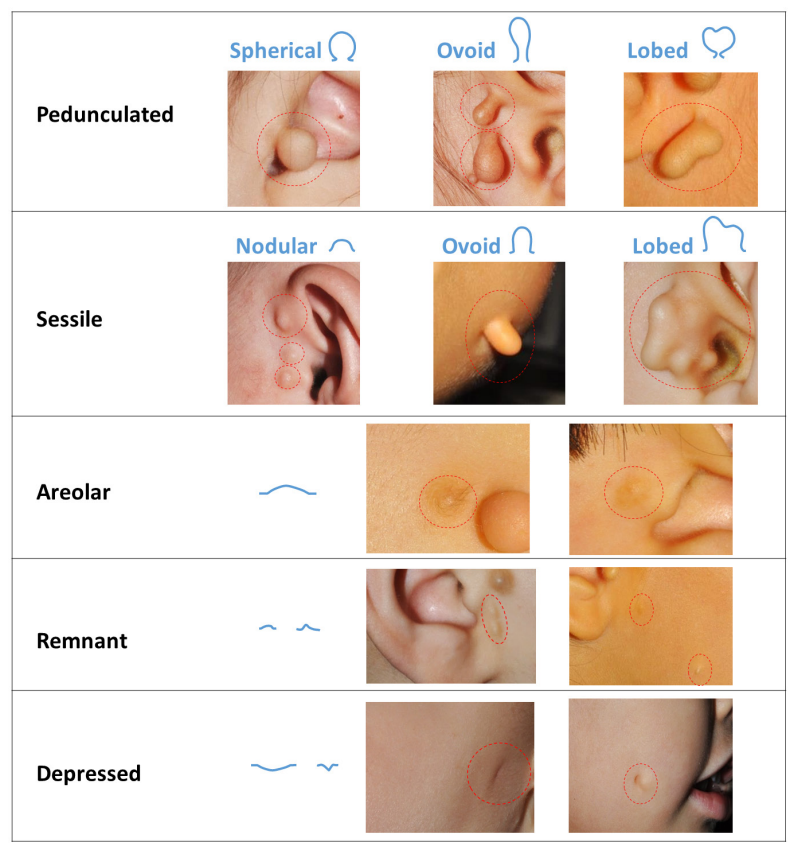

\section{Classification according to protrusion pattern and body shape}

AAs were also classified according to the protrusion pattern above the surrounding surface: pedunculated, sessile, areolar, remnant, or depressed. The pedunculated and sessile patterns were subclassified as spherical, ovoid, lobed, or nodular AAs according to their body shape (Fig. 3). The pedunculated pattern had a neck and spherical, ovoid, or lobed body shape. The sessile pattern did not have a neck and had a nodular, ovoid, or lobed body shape. The areolar pattern was a pigmented and slightly convex lesion in a wide round shape like the nipple areola. The remnant pattern was a tiny and slightly protruding lesion. The depressed pattern was a dimple or pit lesion.

\section{Presence of cartilage root}

Many AAs had a cartilage root at their base. The presence and direction of the cartilage root was identified during surgical excision according to the location, especially in the pedunculated and sessile patterns (Fig. 4).

\section{Fig. 4. Cartilage root of accessory auricle}

The cartilage root of the accessory auricle is denoted by a yellow arrow.

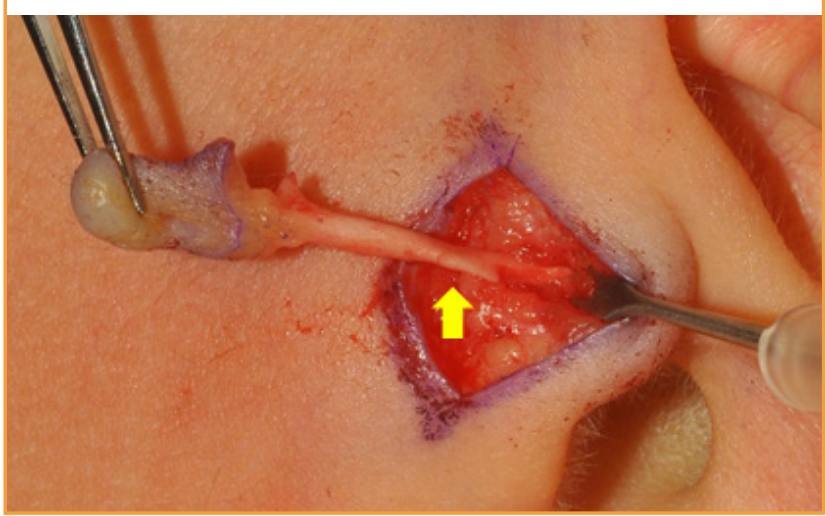

\section{Kyung Hee University coding system}

We propose categorizing AAs by a combination of the anatomical location, protrusion pattern, shape, and cartilage root presence, which can be represented by simple abbreviations as follows: Location is coded as IC (intracrural), IT (intratragal), IL (intralobal), PC (precrural), PTs (superior pretragal), PTm (middle pretragal), PTi (inferior pretragal), PL (prelobal), Ba (anterior buccal), or Bp (posterior buccal). Shape is described by the protrusion pattern and body shape. The protrusion pattern is coded as P (pedunculated), S (sessile), A (areolar), R (remnant), or $\mathrm{D}$ (depressed). The body shape is coded as $\mathrm{S}$ (spherical), $\mathrm{O}$ (ovoid), L (lobed), or $\mathrm{N}$ (nodular). The presence of a cartilage root is coded as + (presence) or - (absence). For example, a superior pretragal subtype and pedunculated ovoid shape with a cartilage root is coded as $\mathrm{PTsPO}+$.

\section{RESULTS}

The total number of AAs in 502 patients was 1,003. They included two helical crus deformities, 158 tragal deformities, and four ear lobe deformities. Three patients had microtia and one had a constricted ear deformity. A family history of AA was present in $11 \%$. The age range for the patients who underwent surgery was 7 days to 30 years. The average number of AAs per patient was two. Data for the patients included in the study are listed in Table 1.

\section{Classification according to location}

Table 2 and Fig. 5 show the classification of AAs according to their locations. Among the facial anatomical units, preauricular AAs were observed in 676 cases (67.4\%), intraauricular AAs in 277 cases (27.6\%), and buccal AAs in 50 cases (5.0\%).

Among the subunits of preauricular AAs, PTs AAs were ob- 
Table 1. Characteristics of 502 patients with 1,003 accessory auricles (2013-2015)

\begin{tabular}{|lc|}
\hline Patient characteristics & No. (\%) \\
\hline Site of deformity & \\
Right & $208(41.4)$ \\
Left & $181(36.1)$ \\
Bilateral & $113(22.5)$ \\
No. of accessory auricles & \\
1 & $203(40.4)$ \\
2 & $169(33.7)$ \\
3 & $74(14.7)$ \\
4 & $43(8.6)$ \\
5 & $11(2.2)$ \\
6 & $1(0.2)$ \\
7 & $1(0.2)$ \\
Sex & \\
Male & $271(54)$ \\
Female & $231(46)$ \\
\hline
\end{tabular}

\section{Fig. 5. Location of accessory auricles}

Among the facial units, preauricular accessory auricles (AAs) were the most common (67.4\%), followed by intraauricular AAs (27.6\%) and buccal AAs (5.0\%). Among the subunits, superior pretragal AAs were most common in the preauricular area, with intratragal AAs in the intraauricular area, and posterior buccal AAs in the buccal area.

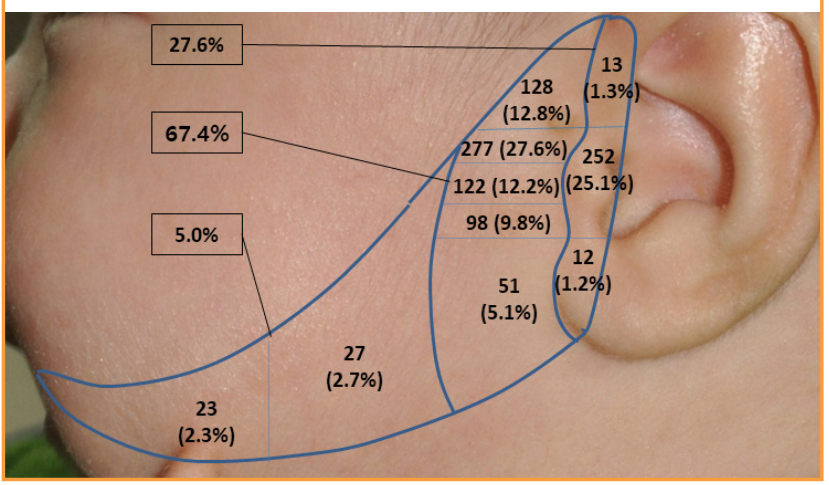

served in 277 of the total cases (27.6\%), making them the most commonly observed preauricular subunit (41.0\%). PC AAs were observed in 128 (12.8\%) cases, PTm AAs in 122 (12.2\%), PTi AAs in 98 (9.8\%), and PL AAs in 51 (5.1\%).

Among the subunits of intraauricular AAs, IT AAs were observed in 252 of the total cases (25.1\%), making them the most commonly observed intraauricular subunit (91.0\%). IC AAs were observed in 13 cases (1.3\%) and IL AAs in 12 (1.2\%).

Among the subunits of buccal AAs, Bp AAs were observed in 27 cases (2.7\%) and Ba AAs in 23 (2.3\%).

\section{Classification according to protrusion pattern and body shape}

Table 3 shows a classification of AAs according to their shapes. Among the total cases, the sessile protrusion pattern was the
Table 2. Classification of 1,003 accessory auricles according to location

\begin{tabular}{|lccc|}
\hline Location & No. of cases & Subtotal (\%) & Total (\%) \\
\hline Preauricular & 676 & 100 & 67.4 \\
Precrural & 128 & 18.9 & 12.8 \\
Pretragal & 497 & 73.5 & 49.6 \\
Superior pretragal & 277 & 41.0 & 27.6 \\
Middle pretragal & 122 & 18.0 & 12.2 \\
Inferior pretragal & 98 & 14.5 & 9.8 \\
Prelobal & 51 & 7.5 & 5.1 \\
Intraauricular & 277 & 100 & 27.6 \\
Intracrural & 13 & 4.7 & 1.3 \\
Intratragal & 252 & 91.0 & 25.1 \\
Intralobal & 12 & 4.3 & 1.2 \\
Buccal & 50 & 100 & 5.0 \\
Anterior buccal & 23 & 46.0 & 2.3 \\
Posterior buccal & 27 & 54.0 & 2.7 \\
Total & 1,003 & & 100 \\
\hline
\end{tabular}

Table 3. Classification of 1,003 accessory auricles according to protrusion pattern and body shape

\begin{tabular}{|lcccc|}
\hline \multirow{4}{*}{ Shape } & \multicolumn{4}{c|}{ No. of cases (\%) } \\
\cline { 2 - 5 } & Preauricular & Intraauricular & Buccal & Total \\
\hline Pedunculated & $300(44.4)$ & $39(14.1)$ & $31(62)$ & $370(36.9)$ \\
Spherical & $102(15.1)$ & $18(6.5)$ & $12(24)$ & $132(13.2)$ \\
Ovoid & $188(27.8)$ & $21(7.6)$ & $14(28)$ & $223(22.2)$ \\
Lobed & $10(1.5)$ & 0 & $5(10)$ & $15(1.5)$ \\
Sessile & $276(40.8)$ & $223(80.4)$ & $5(10)$ & $504(50.2)$ \\
Nodular & $128(18.9)$ & $58(20.9)$ & $3(6)$ & $189(18.8)$ \\
Ovoid & $103(15.2)$ & $30(10.8)$ & $2(4)$ & $135(13.5)$ \\
Lobed & $45(6.7)$ & $135(48.7)$ & 0 & $180(17.9)$ \\
Areolar & $27(4.0)$ & $0(0)$ & $2(4)$ & $29(2.9)$ \\
Remnant & $70(10.4)$ & $15(5.4)$ & $4(8)$ & $89(8.9)$ \\
Depressed & $3(0.4)$ & $0(0)$ & $8(16)$ & $11(1.1)$ \\
Subtotal (\%) & 100 & 100 & 100 & \\
Total & $676(67.4)$ & $277(27.6)$ & $50(5.0)$ & $1,003(100)$ \\
\hline
\end{tabular}

most common, with 504 (50.2\%) AAs. In addition, there were 370 (36.9\%) pedunculated AAs, 29 (2.9\%) areolar AAs, 89 (8.9\%) remnant AAs, and 11 (1.1\%) depressed AAs.

There were $300(44.4 \%)$ pedunculated AAs in the preauricular area, accounting for the most common protrusion pattern. In addition, there were 276 (40.8\%) sessile AAs, 27 (4.0\%) areolar AAs, 70 (10.4\%) remnant AAs, and three (0.4\%) depressed AAs. The pedunculated pattern included 188 AAs (27.8\%) with an ovoid shape, accounting for the most common shape of the pedunculated pattern. In addition, there were 102 AAs (15.1\%) with a spherical shape and $10 \mathrm{AAs}(1.5 \%)$ with a lobed shape. Among the sessile AAs, 128 (18.9\%) had a nodular shape, accounting for the most common shape of the sessile pattern. In addition, there were 103 AAs (15.2\%) with an ovoid shape and 45 AAs (6.7\%) with a lobed shape. 
Table 4. Cartilage root presence according to location in the pedunculated and sessile patterns

\begin{tabular}{|lc|}
\hline Location & Presence rate (\%) \\
\hline Intracrural & 100 \\
Intratragal & 88.7 \\
Intralobal & 0 \\
Precrural & 100 \\
Superior pretragal & 96 \\
Middle pretragal & 65 \\
Inferior pretragal & 36 \\
Prelobal & 2 \\
Buccal & 75 \\
\hline
\end{tabular}

In the intraauricular area, there were 232 (80.4\%) sessile AAs, accounting for the most common protrusion pattern. In addition, there were 39 (14.1\%) pedunculated AAs and 15 (5.4\%) remnant AAs. Among the sessile AAs, 135 (48.7\%) had a lobed shape, which was the most common shape of the sessile pattern. Tragal deformities were classified according to their shape. Most of the tragal deformities were classified as sessile lobed. Thus, many of the sessile lobed AAs were actually tragal deformities. In addition, there were 58 AAs (20.9\%) with a nodular shape and $30 \mathrm{AAs}(10.8 \%)$ with an ovoid shape. Among the pedunculated AAs, 21 (7.6\%) had an ovoid shape, accounting for the most common shape of the pedunculated pattern. In addition, there were $18 \mathrm{AAs}(6.5 \%)$ with a spherical shape.

In the buccal area, there were 31 (62\%) pedunculated AAs, accounting for the most common protrusion pattern. In addition, there were five (10\%) sessile AAs, two (4\%) areolar AAs, four (8\%) remnant AAs, and eight (16\%) depressed AAs. Among the pedunculated pattern, 14 AAs $(28 \%)$ had an ovoid shape, accounting for the most common shape of the pedunculated pattern. In addition, there were 12 AAs (24\%) with a spherical shape and five AAs (10\%) with a lobed shape. In the sessile pattern, there were three AAs (6\%) with a nodular shape, accounting for the most common shape of the sessile pattern. In addition, there were two AAs (4\%) with an ovoid shape.

\section{Cartilage root presence}

A cartilage root was present in $78.4 \%$ of AAs. In the pedunculated and sessile patterns, the presence of the cartilage root was $100 \%$ in the intracrural area, $88 \%$ in the intratragal area, $0 \%$ in the intralobal area, $100 \%$ in the precrural area, $96 \%$ in the superior pretragal area, $65 \%$ in the middle pretragal area, $36 \%$ in the inferior pretragal area, $2 \%$ in the prelobal area, and $75 \%$ in the buccal area. Table 4 and Fig. 6 show cartilage root presence by location in AAs of the pedunculated and sessile patterns.

\section{Fig. 6. Cartilage root presence according to location}

Cartilage root presence varied according to location in the pedunculated and sessile patterns. In the pedunculated and sessile patterns, a cartilage root was present in $100 \%$ of intracrural cases, $88 \%$ of intratragal cases, $0 \%$ of intralobal cases, $100 \%$ of precrural cases, $96 \%$ of superior pretragal cases, $65 \%$ of middle pretragal cases, 36\% of inferior pretragal cases, $2 \%$ of prelobal cases, and $75 \%$ of buccal area cases.

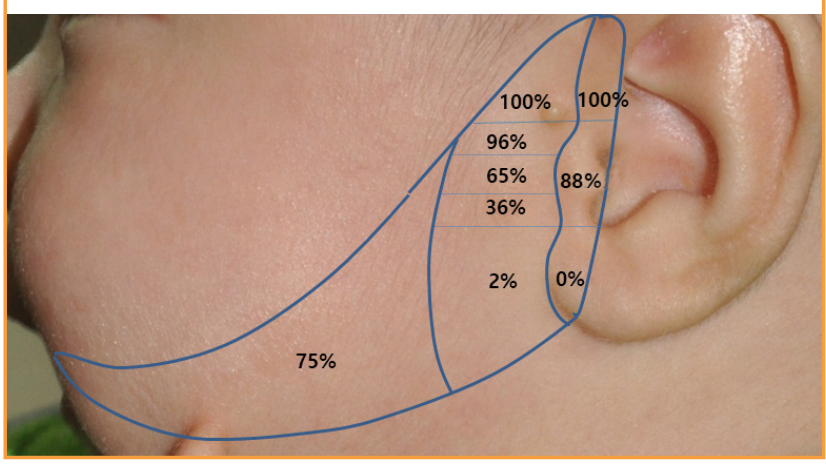

\section{DISCUSSION}

AAs are abnormal appendages of the anterior auricle that persist during early embryonic development. Embryonic auricular components appear early in the fourth week of gestation. Auricular components develop at the side of the mouth and gradually move laterally and dorsally during development $[1,2]$. The location of AAs is correlated with the course of embryonic development of the three hillocks of the first pharyngeal arch. Therefore, abnormal developmental appendages may be located within a curved triangle from the oral commissure groove to the anterior auricle between the helical spine and the ear lobe (Fig. 1). These locations correlated with our findings of the distribution of AAs. Several other studies have described these abnormal skin appendages as preauricular tags [5,6]. Those studies regarded other skin appendages that were not located in the preauricular area as variants of AAs. However, these skin appendages may be present at the buccal or intraauricular area. Our study data showed that buccal AAs were present in 5.0\% of cases and intraauricular AAs in 27.6\%. Other studies have regarded skin appendages as accessory tragi $[3,4]$. Our study showed that $73.5 \%$ of preauricular AAs and $91.0 \%$ of intraauricular AAs were found in the tragus region. Most AAs were located in the tragus region and had the potential to develop into a tragus. They could be called accessory tragi. However, precrural, prelobal, crural, and lobal AAs were also present and might not have had the potential to develop into a tragus. They might have had the potential to develop into the crus or ear lobe. Some studies have described these skin appendages as AAs $[7,8]$, and we also propose that $\mathrm{AA}$ is the most proper term for these ab- 
normal skin appendages. Pretragal and intratragal were the most common subtypes in the preauricular and intraauricular area. Pretragal and intratragal AAs might be fated to form the tragus and may have the same origin as the embryonic hillock. Park [9] described an entity called "hillock 2," which might be the most common component of these malformations. Interestingly, we observed that many pretragal or intratragal AAs were grouped in pairs, while most AAs at other areas were of a single type. Further embryological study is required to explain this observation.

AA shape was classified according to the protrusion pattern above the surface as pedunculated, sessile, areolar, remnant, or depressed. Pedunculated and sessile patterns were subclassified as spherical, ovoid, lobed, or nodular according to their body shapes. Most AAs (81.7\%) were sessile or pedunculated. Areolar, remnant, and depressed patterns were less common accounting for $12.9 \%$ of the. These minor patterns were also developmental anomalies of the three hillocks of the first pharyngeal arch. During the process of forming the anterior auricular structure, an unknown obstacle might cause the hillocks to form a protrusion, depression, or other morphologic anomaly. We believe that AAs can be described well following these patterns and shapes.

The distribution of AAs according to location and shape observed in our center (Tables 2 and 3 ) may differ from the distribution in the general population. Our results may have been biased because our center specializes in performing tragal deformity revision surgery. Our study population included more tragal deformities than are present in the general population. In our classification, tragal deformities were classified according to shape. Most of those anomalies were classified as sessile lobed and intratragal AAs. Thus, in a general population of individuals with an AA, the shape distribution may be similar to that in our preauricular or buccal area cases. The pedunculated pattern was the most common in the preauricular (44.4\%) and buccal (62\%) areas. An ovoid and pedunculated or sessile pattern was the most common shape in the preauricular (43\%) and buccal (32\%) areas. In addition, in a general population of individuals with an AA, the intratragal subtype (25.1\%) may be substantially less common than in our study population, and the pretragal subtype (49.6\%), especially the superior pretragal subtype (27.6\%), may be much more common.

Most AAs have a cartilage root. The rate of cartilage root presence was $78.4 \%$. Each hillock of the first pharyngeal arch develops into each component of the anterior auricle. The helical crus and tragus have a cartilage structure base, while the ear lobe does not. We presumed that the presence of a cartilage root in an AA depends on the location, and analyzed the presence ac- cordingly. We assumed that an embryonic hillock with the potential to develop into the helical crus or tragus requires a cartilage root and that a hillock with the potential to develop into an ear lobe may not require a cartilage root. Among pedunculated and sessile patterns, all precrural and intracrural subtypes had cartilage roots, but $2 \%$ of prelobal and $0 \%$ of intralobal subtypes had a cartilage root. Prelobal or intralobal AAs might be fated to develop into an ear lobe, meaning that a cartilage base would be unnecessary. Interestingly, $96 \%$ of superior pretragal, $65 \%$ of middle pretragal, and $36 \%$ of inferior pretragal subtypes had a cartilage root. The superior portion of pretragal AAs might have a high likelihood of developing into the helical crus or tragus, with a cartilage base, whereas the inferior portion of pretragal AAs might have a high likelihood of developing into the ear lobe, which does not have a cartilage base. Whether the AA was located in the buccal, preauricular, or intraauricular area, the cartilage root was directed toward the position of the tragohelical sulcus or tragus of the auricle, which the embryonic hillock may form. In addition, we observed many pretragal or intratragal AAs that did not have a cartilage root, but instead had a fibrotic band directed toward the helical crus or tragus.

In conclusion, in terms of location, AAs were classified as intraauricular, preauricular, and buccal. Intraauricular AAs were subdivided into intracrural, intratragal, and intralobal types. Preauricular AAs were subdivided into precrural, superior pretragal, middle pretragal, inferior pretragal, and prelobal types. Buccal AAs were subdivided into anterior buccal and posterior buccal types. In terms of protrusion pattern, AAs were classified as pedunculated, sessile, areolar, remnant, and depressed. Pedunculated and sessile patterns were subclassified as having spherical, ovoid, lobed, and nodular shapes. The most common location subtype was superior pretragal (41.0\%) in the preauricular area, intratragal (91.0\%) in the intraauricular area, and posterior buccal (54.0\%) in the buccal area. The most common protrusion pattern and shape was pedunculated ovoid in the preauricular (27.8\%) and buccal (28.0\%) areas, and sessile lobed in the intraauricular (48.7\%) area. Cartilage root presence was dependent on the location. Regarding location and shape, the most common type of preauricular AA was the superior pretragal pedunculated ovoid AA (13.2\%) with a cartilage root, the code for which was $\mathrm{PTsPO}+$. The KHU coding system may prove particularly useful in communicating the location, shape, and cartilage presence of AAs. Although the study was limited by the fact that all data were from one institution, we expect that this new classification and coding system could provide a framework for management of AAs. 


\section{NOTES}

\section{Conflict of interest}

No potential conflict of interest relevant to this article was reported.

\section{Ethical approval}

The study was performed in accordance with the principles of the Declaration of Helsinki. Written informed consents were obtained.

\section{Patient consent}

The patients provided written informed consent for the publication and the use of their images.

\section{ORCID}

Jungil Hwang https://orcid.org/0000-0001-6482-3696

\section{REFERENCES}

1. Kagurasho M, Yamada S, Uwabe C, et al. Movement of the external ear in human embryo. Head Face Med 2012;8:2.
2. Porter CJ, Tan ST. Congenital auricular anomalies: topographic anatomy, embryology, classification, and treatment strategies. Plast Reconstr Surg 2005; 115:1701-12.

3. Brownstein MH, Wanger N, Helwig EB. Accessory tragi. Arch Dermatol 1971;104:625-31.

4. Bahrani B, Khachemoune A. Review of accessory tragus with highlights of its associated syndromes. Int J Dermatol 2014;53:1442-6.

5. Kugelman A, Hadad B, Ben-David J, et al. Preauricular tags and pits in the newborn: the role of hearing tests. Acta Paediatr 1997;86:170-2.

6. Dancel R, Price D, Kaufmann L. Evaluation of newborns with preauricular skin lesions. Am Fam Physician 2012;85: 993-8.

7. Beder LB, Kemaloglu YK, Maral I, et al. A study on the prevalence of accessory auricle anomaly in Turkey. Int J Pediatr Otorhinolaryngol 2002;63:25-7.

8. Moon IY, Oh KS. Surgical correction of an accessory auricle, polyotia. Arch Plast Surg 2014;41:427-9.

9. Park C. Lower auricular malformations: their representation, correction, and embryologic correlation. Plast Reconstr Surg 1999;104:29-40. 